\title{
Modelling Diffusion in a Physically Constrained System: A Numerical Approach
}

\section{AUTHORS: Adam Jozefiak, Jim Zhang Hao Li \\ SCHOOL: University of British Columbia}

\section{Abstract}

Diffusion has been described on a microscopic scale by Einstein as a probabilistic collision of particles. On a macroscale, diffusion has been thoroughly described by Fick's laws. However, the solutions to Fick's laws are limited to idealized physical systems. The aim of this experimental study is to provide a mathematical model for diffusion which incorporates both macroscopic and microscopic properties to effectively model diffusion in a geometrically constrained two-dimensional system. Based on macroscopic and microscopic properties, two-dimensional diffusion was modelled as a summation of equally probable paths of diffusion. The point source diffusion of hydrochloric acid in an arena with variable barrier dimensions was monitored continuously using a pH probe. The numerical solution of the mathematical model for each experimental condition was determined and the pre-exponential factor was fit to the measurements. The average pre-exponential value was determined for each experimental condition, and t-scores were calculated to compare the average pre-exponential values which were found to be statistically similar. This indicates that the proposed model is an accurate model as it predicts identical pre-exponential values between experimental conditions, accounting for all variants that it attempts to model. This model provides a bridge between the microscopic and macrcoscopic theoretical descriptions of diffusion that were independently postulated by Einstein and Fick. Applications of the model include the approximation of locations of leakage in hydraulic systems.

\section{Key Words}

Modelling Diffusion, Numerical Computation, Ficks Laws

\section{Introduction}

Adolf Fick, in 1855, provided a mathematical explanation of diffusion by postulating two fundamental laws for diffusion, known as Fick's laws (1).

Fick's first law states that the flux of diffusion - the movement of particles through a given area - is proportional to the diffusive constant multiplied by the negative of the concentration gradient (1). The differential equation is described as

$$
J=-D \frac{d C}{d x}
$$

Where $\mathrm{J}$ represents the diffusive flux $\left(\mathrm{mol} / \mathrm{m}^{2} \mathrm{~s}\right), \mathrm{C}$ is the concentration in $\left(\mathrm{mol} / \mathrm{m}^{3}\right), x$ is some defined position along a defined $\mathrm{x}$-axis in $(\mathrm{m})$, and the $D$, the diffusion constant in $\left(\mathrm{m}^{2} / \mathrm{s}\right)$ which describes a solute's relative rate of diffusion in relation to the properties of the solute, solvent, and overall system $(1,2)$.

Fick's second law of diffusion states that the change in concentration in some defined space with respect to time is proportional to the second derivative of concentration with respect to position multiplied by the diffusion constant (1). This law states that a concentration gradient will result in concentration changes in the system, where regions down a concentration gradient will experience an increasing concentration with time. The partial differential equation is described as

$$
\frac{d C}{d t}=D \frac{d^{2} C}{d x^{2}}
$$

Although Fick's laws of diffusion provide a thorough description of diffusion, they are limited to a macroscopic explanation of the phenomenon. It was later that Einstein described the observed Brownian Motion as a series of stochastic collisions between particles, thus bridging the kinetic molecular theory of heat with classical thermodynamics (2).

By this stochastic nature of particle collisions, individual particles have an equal probability of moving in any direction (2). Secondly, in an arbitrarily defined three-dimensional coordinate system, movements along an axis are independent of movements along the other two axes (2).

Many solutions to Fick's laws of diffusion are limited to idealized systems and consequently fail to account for more complex geometries of the physical system. In response, this study investigates the feasibility of the development of a more holistic model of diffusion that incorporates complex geometries of a physical system. An idealized system in this context is defined as a physical system of perfect geometric dimensions such a line, rectangle, or a cylinder.

The model developed in this study will provide a new bridge between the macroscopic and microscopic descriptions of diffusion 
that have been developed independently by Einstein and Fick. Furthermore, the model will be a basis for future models that can be applied to macroscopic scenarios of fluid diffusion. This has pertinence in several fields, including but not limited to industrial, environmental, and health care settings.

To achieve the purpose of this study, a new numerical model of diffusion based on solutions to Fick's laws and the microscopic properties of diffusion is postulated and verified against experimental results from a physically constrained two-dimensional system of diffusion.

\section{Methods}

Development of the Model.As a basis for this new model, the solution to a one-dimensional idealized system described by Fick's second law is employed (3). The solution is

$$
C(r, t)=\frac{A}{\sqrt{t}} e^{\frac{-r^{2}}{4 D t}}
$$

where $A$ is a dimension-less preexponential factor, $t$ is time in (s), $r$ is displacement in (m). The pre-exponential factor remains constant for a specific system, and is an empirically determined value (3).

The diffusion constant for the experimental system is approximated using the Einstein- Stokes equation

$$
D=\frac{K_{B} T}{6 \pi P R}
$$

Where, $K_{B}$, the Boltzmann constant is $1.38065 \times 10^{-23} \mathrm{~m}^{2} \mathrm{~kg} / \mathrm{s}^{2} \mathrm{k}$, $T$ is the absolute temperature of the system in $(\mathrm{K}), P$, is the dynamic viscosity of the solvent in $\mathrm{Ns} / \mathrm{m}^{2}$, and $R$ is the solute's radius in $(\mathrm{m})$ $(2,4)$.

Figure 1 displays the idealized onedimensional system that equation 3 represents and as well as the two-dimensional systems of diffusion that this study investigates.

To model a two-dimensional physically constrained system, diffusion is treated as a summation of all possible and reasonable net paths a particle could take to the final displacement position. A reasonable path of diffusion is defined as a path with as few directional changes as possible, and whereby all paths should contain the same number of directional changes with the exception of a few paths that may have fewer directional changes as is the case with the central path of the second diagram in Figure 1.

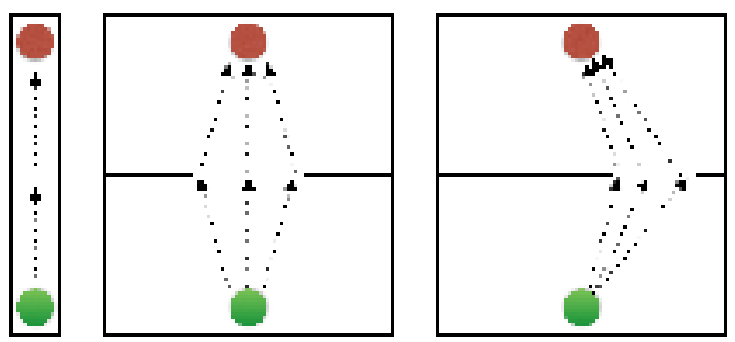

Figure 1: Representations of Fick's one-dimensional sytem of diffusion, and two-dimensional systems.

Green dots represent initial points of concentration and red dots represent final postions after displacement; arrows represent paths of net diffusion.

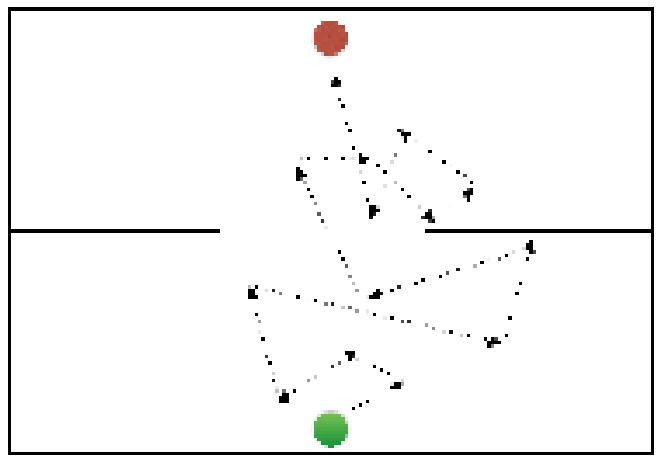

Figure 2: Representations of an unreasonable path of diffusion.

Figure 2 displays an unreasonable path. Although this path is a realistic representation of an individual particle's stochastic displacement (2), on a macroscale Fick's laws treat these stochastic movements in aggregate by quantifying the net diffusive flux rather than the exact movement of a particle $(1,3)$. The assumption with choosing only paths defined as reasonable paths as opposed to including unreasonable paths in this model lies in the statistical improbability of individual particles moving in identical, or at least similar, stochastic paths like in Figure 2. It is more likely that particles would move in the reasonable paths, or at least paths that more closely resemble them, as compared to the stochastic path displayed in Figure 2. Further, an addition of more paths, with changes in their direction at multiple and different points than just at the barrier's slit would have led to an increasingly large number of computational operations to solve the model, which would have become overly cumbersome for the scope of this study. Consequently, equation 3 provides an effective solution to an idealized one dimensional system of diffusion and by using this solution as the basis for the summation of many paths of diffusion, the same assumption about the net diffusion along the paths should also apply.

In this new model, each path is weighed by the angle the path 
represents as a proportion of the overall angle of diffusion. By this view, an infinite number of net paths of diffusion would result in an infinitesimally small weighing of each path. The key assumption of this two-dimensional model is the microscopic property that particles have an equal probability in moving in any direction (2). By the use of an equally probable diffusion with respect to angle, each path must be equally spaced apart in terms of an internal angle $(\mathrm{d} \theta)$ between paths of diffusion from an initial concentration source.

Another reason why paths were chosen to be weighted with respect to angle was because of the empirical trend noted when slit size and position were varied. It was found that the experimental trends correlated with changes in the size of the angle between the slit edges and the central angle as opposed to the changes in the absolute size of the slit. This is evidenced by the more drastic change in experimental results between the $1.0 \mathrm{~cm}$ and $2.0 \mathrm{~cm}$ slit trials versus the difference in the results between the $10.0 \mathrm{~cm}$ slit and a no barrier (21.2 cm slit) trials. Likewise, experimental data showed that the positioning of a same size slit $(2.0 \mathrm{~cm})$ with respect to a central angle altered the diffusive rate, even though the slit width remained constant. Thus, these empirical observations, along with the theoretical assumptions about equal probabilistic diffusion into any direction led to the assumption of weighing reasonable paths of diffusion with respect to angle.

Thus, this summation of paths, each weighed by $d \theta$, is

$$
C(r, t)=\sum_{i=0}^{N} d \theta \times \frac{A}{\sqrt{t}} e^{\left(\frac{-\left(r_{i}\right)^{2}}{4 D t}\right)}
$$

Where $\mathrm{N}$ is defined as

$$
N=\frac{\theta}{d \theta}
$$

Since the displacement path distance $r_{\mathrm{i}}$ is being incremented with respect to the central angle, $r_{\mathrm{i}}$ becomes a function of the incremented angle where

$$
r_{i}=R\left(\theta_{0}+d \theta \times i\right)
$$

In order to computationally solve equation 4 , a function $R$ must be developed where the displacement distance $r_{\mathrm{i}}$ can be determined repetitively in increments, with respect to a constant change of the central angle (Equation 7). Figures 3 and 4 display geometries of two-dimensional diffusion systems with incremented $r_{\mathrm{i}}$ displacements. The systems displayed in these figures are identical to this study's experimental conditions, and for the purpose of this study, $r_{\mathrm{i}}$ was solved for using standard Pythagorean and trigonometric relationships.

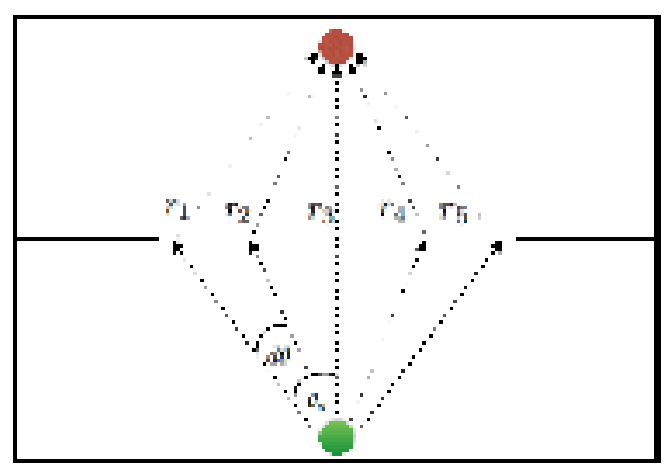

Figure 3: Representation of the summation of many paths diffusion model.

A barrier bisects the system through the centre, containing a permeable opening along a central axis.

\section{Cer.trul Axis}

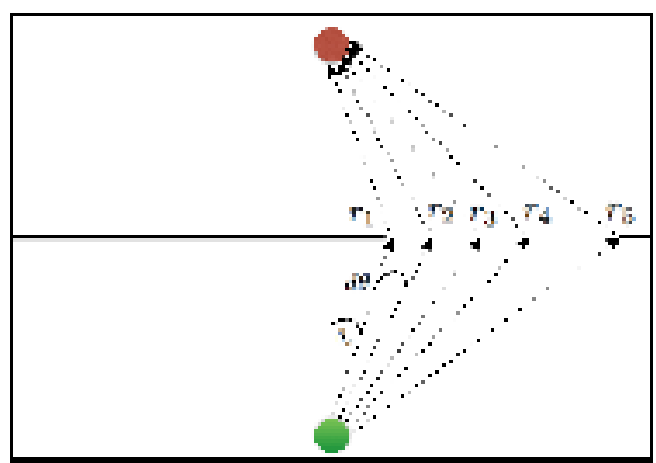

Figure 4: Representation of the summation of many paths diffusion model in a system with a shifted barrier opening away from the central axis.

\section{Experimental Procedure}

Hydrochloric acid was chosen as the diffusion particle due to its ability to induce an observable $\mathrm{pH}$ change. All trials were conducted in a glass experimental arena measuring $21.2 \mathrm{~cm} \times 25.4 \mathrm{~cm} \times 7$ $\mathrm{cm}$. A corrugated-plastic barrier with variable-sized slits bisects the arena into two halves as shown in Figures 5 and 6 . The medium for diffusion throughout the experiment was $700 \mathrm{~mL}$ of tap water. The relatively shallow water depth of about $1.25 \mathrm{~cm}$ could be used as an effective model for two dimensional diffusion as the movement of particles in the two-dimensional horizontal plane is independent of vertical-depth movements (2). In each trial, $10.0 \mathrm{~mL}$ of $8.62 \mathrm{M}$ hydrochloric acid was injected into a small closed plastic container via a serological syringe, which was subsequently secured on the arena edge facing towards the barrier. A Vernier $\mathrm{pH}$ probe secured on the opposite side was used to record the $\mathrm{pH}$ data over time. Changes in $\mathrm{pH}$ during the diffusion process were assumed to be due to the 
diffusion of hydrochloric acid and hydronium.

The $\mathrm{pH}$ probe was set to start recording data when the plastic container was opened. Experimental trials were performed for a minimum duration of 500 seconds, with the exception of the positive control which lasted for 480 seconds. Extraneous environmental variables such as temperature and air flow were minimized through conducting the experiment in the same indoor laboratory setting. Furthermore, the $\mathrm{pH}$ probe was calibrated after every experiment by placing it in a buffer solution.

Table 1: List of experimental treatments and their angular and dimensional parameters.

\begin{tabular}{llr}
\hline Treatment & $\begin{array}{l}\text { Slit Size } \\
\text { Angles between Slit } \\
\text { Edges and Axis } \\
\theta_{l} \text { and } \theta_{r} \\
\text { (degrees) }\end{array}$ & $\begin{array}{r}\text { N/A } \\
(\mathrm{cm})\end{array}$ \\
\hline 1 & $0 \mathrm{~cm}$ & \\
2 & $21.2 \mathrm{~cm}$ & $41.15^{\circ}$ and $-45.15^{\circ}$ \\
3 & $1.0 \mathrm{~cm}$ & $2.25^{\circ}$ and $-2.25^{\circ}$ \\
4 & $2.0 \mathrm{~cm}$ & $4.50^{\circ}$ and $-4.50^{\circ}$ \\
5 & $4.0 \mathrm{~cm}$ & $17.48^{\circ}$ and $-17.48^{\circ}$ \\
6 & $10.0 \mathrm{~cm}$ & $21.49^{\circ}$ and $-21.49^{\circ}$ \\
7 & $2.0 \mathrm{~cm}$ & $15.00^{\circ}$ and $23.05^{\circ}$ \\
8 & $2.0 \mathrm{~cm}$ & $35.00^{\circ}$ and $40.62^{\circ}$ \\
\hline
\end{tabular}

To determine the effects of different slit widths and distances travelled by the diffusing particles, the widths of the slit and the angle that the slit's edges make with the bisecting axis of the arena was varied as shown in Table 1. Each experimental treatment was limited to a minimum of three experimental trials to allow for the measurement of many treatments as opposed to a precise focus on a relatively few number of treatments. An example of the experimental system for treatments 3-6 is represented in Figure 5 while an example of an experimental system for treatments 7 and 8 is represented in Figure 6.

The first trial was performed as a negative control to determine whether or not the plastic barrier was effective in blocking the acid from crossing into the detection region. In addition, to confirm that the acid is able to diffuse in water and induce a drop in $\mathrm{pH}$ in the detection region, a positive control with no barriers was conducted.

For each treatment, a weighted chi-square optimization was performed for the pre- exponential factor $A$ to fit the model function (Equation 4) to the data. The average standard deviation between the measurements within a certain treatment was used as the uncertainty when calculating weighted chi-square values for that treatment. This allows for the comparison of A values to confirm if Fick's second law still holds true in a geometrically constrained system.

To quantify the model's effectiveness in modeling two-dimensional diffusion, a t-score analysis was employed to compare all treatments except for the negative control.

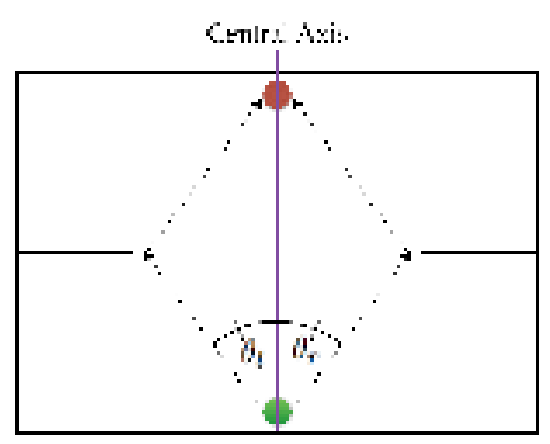

Figure 5: Representation of an experimental arena with the barrier's slit directly between the $\mathrm{pH}$ probe and insertion container.

The green dot represents the position of the insertion container and the red dot represents the position of the $\mathrm{pH}$ probe.

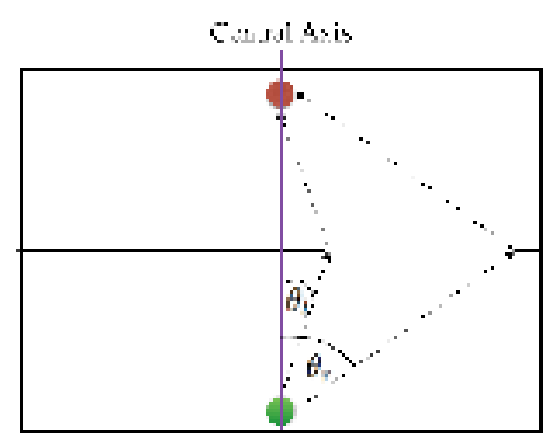

Figure 6: Representation of the experimental arena with the barrier's slit shifted away from the central axis running between the $\mathrm{pH}$ probe and the insertion container.

The green dot represents the position of the insertion container and the red dot represents the position of the $\mathrm{pH}$ probe. 


\section{Results}

Determining the Diffusion Coefficient.The diffusion constant was approximated using the Einstein-Stokes equation (Equation 4) to a value of $2.94 \times 10^{-9}\left(\mathrm{~m}^{2} / \mathrm{s}\right)$.

The values used to calculate this constant are $K_{B}$, the Boltzmann constant, $T$, the absolute temperature of the system, which is $296 K$, $P$, the dynamic viscosity of the aqueous solvent, which is $0.844 \mathrm{x}$ $10^{-3}\left(\mathrm{Ns} / \mathrm{m}^{2}\right)$, and $R$, the solute radius, which is $1.01 \times 10^{-10} \mathrm{~m}$, an average value between the bond distance of a hydrochloric acid molecule and a hydronium molecule (5).

Analyses of Results. Comparing the fitted models, a p-value greater than the conventional 0.05 was found for all trials, and a $p$ value greater than the conventional 0.95 was found for 23 out of the 25 trials. In general, this implies excellent agreement between the data and the fitted models, implying the feasibility of using a numerical approach to approach similar problems in practical settings. For a detailed table of the tabulated chi-square values, refer to the appendix.

The $\mathrm{pH}$ measurements for each set of trials along with the fitted model of the average pre-exponential factor (equation (5)) are reproduced in Figures 7 through 14.

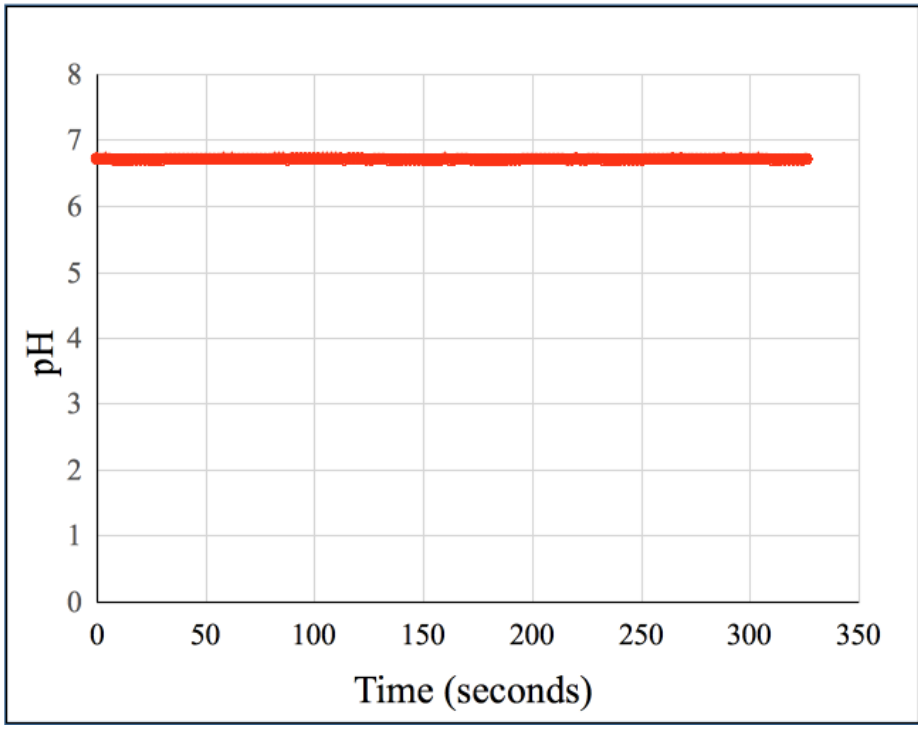

Trial 1

Figure 7: Plot of continuous $\mathrm{pH}$ measurements in two second time intervals in the $0.0 \mathrm{~cm}$ barrier slit treatment (negative control).

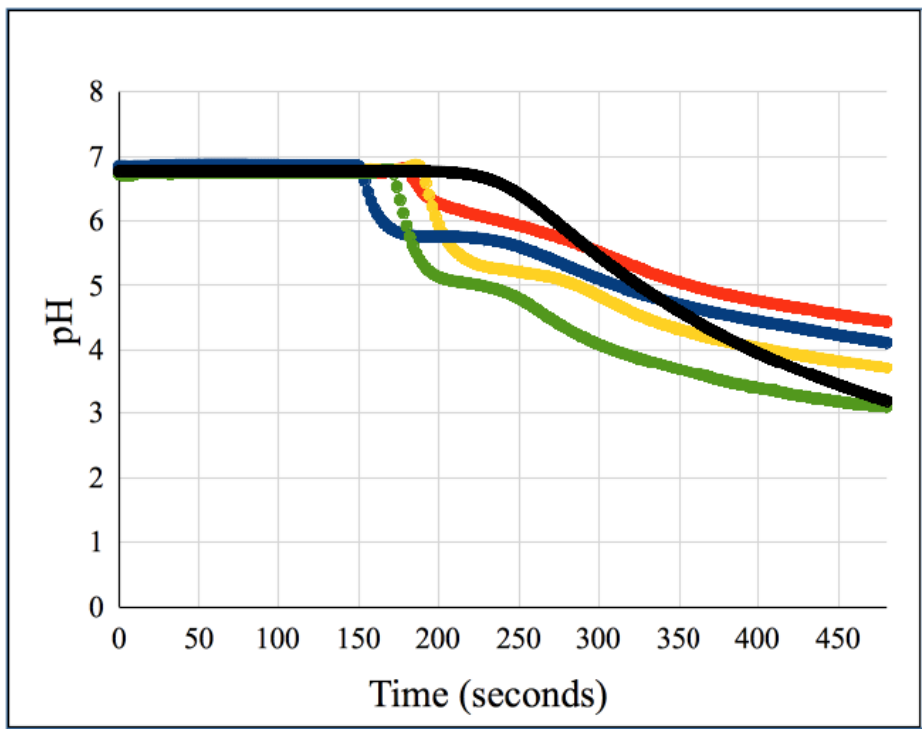

Figure 8: Plot of continuous pH measurements in Model two second time intervals in the $21.2 \mathrm{~cm}$ barrier slit Trial 1 treatment (positive control) with the average fitted Trial 2 model for the treatment.

Trial 3

Trial 4

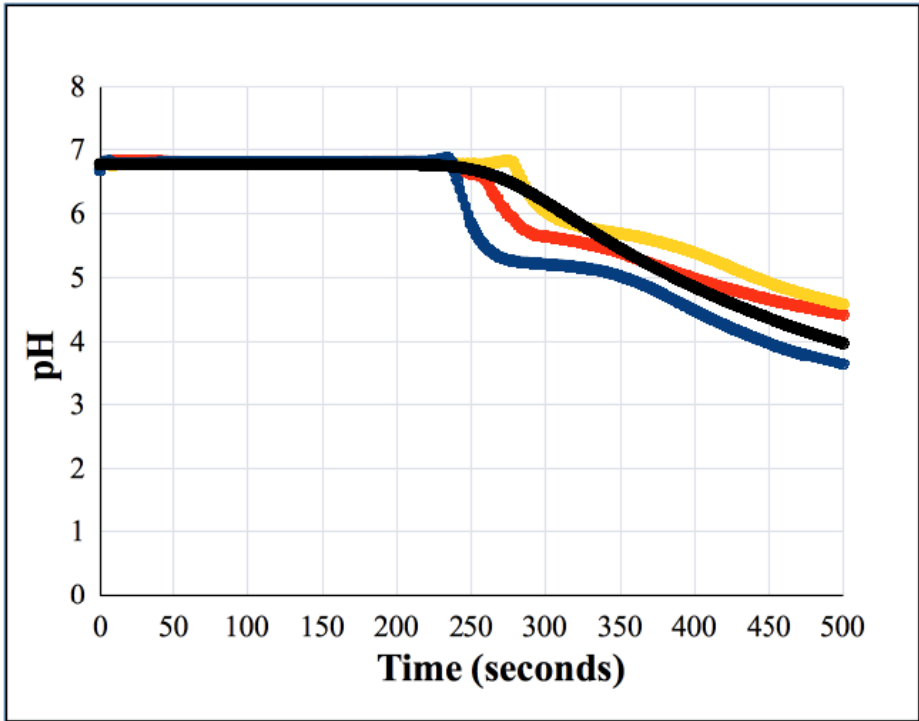

Mode

Trial 1

Trial 2

Trial 3
Figure 9: Plot of continuous pH measurements in two second time intervals in the direct $1.0 \mathrm{~cm}$ barrierslit treatment with the average fitted model for the treatment. 


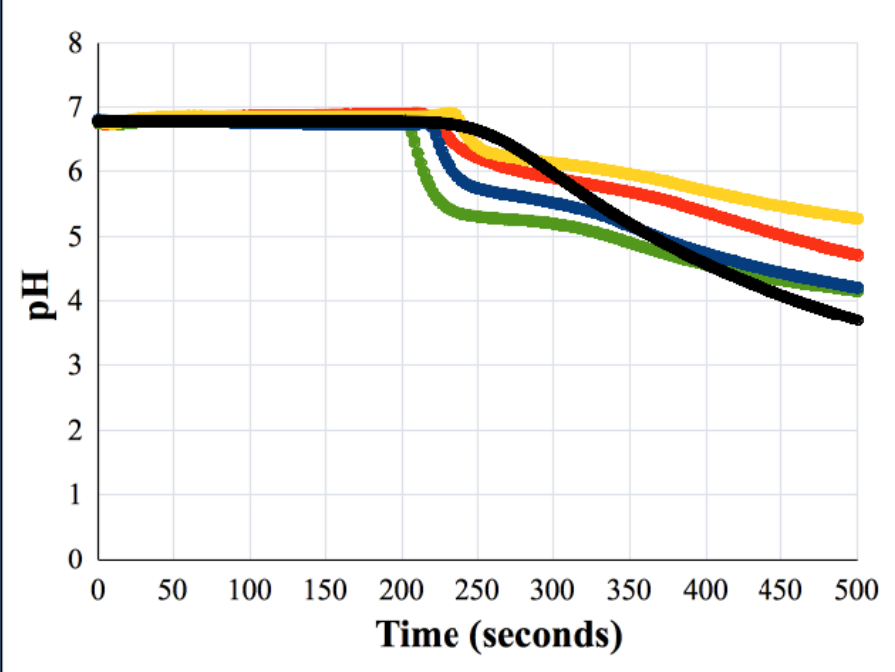

Figure 10: Plot of continuous $\mathrm{pH}$ measurements in two second time intervals in the direct $2.0 \mathrm{~cm}$ barTrial 1 rier slit treatment with the average fitted model for the Trial 2 treatment.

Trial 3

Trial 4

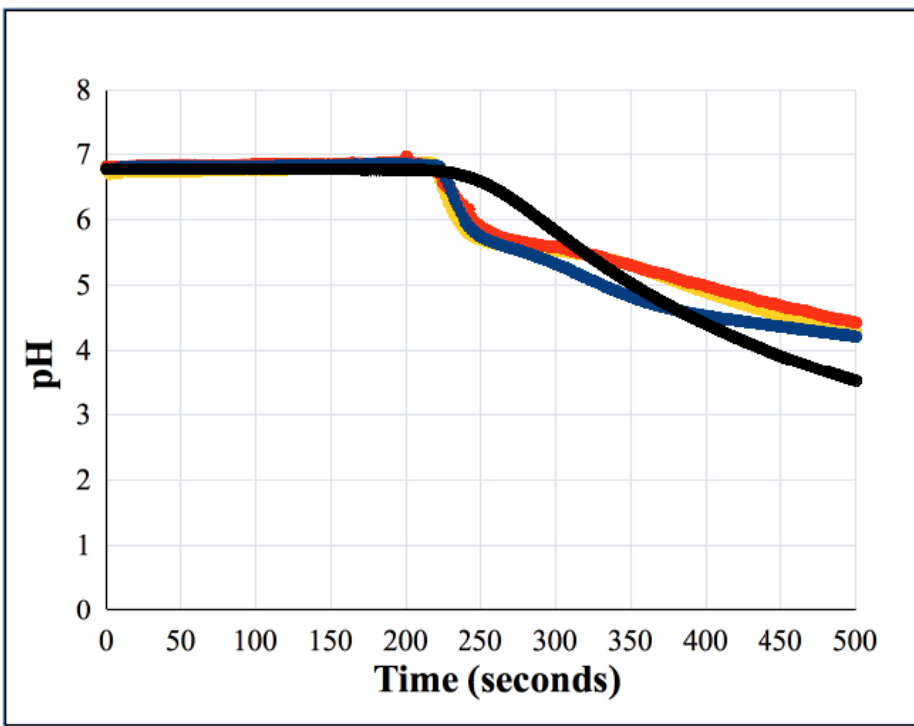

Figure 11: Plot of continuous $\mathrm{pH}$ measurements Model in two second time intervals in the direct $4.0 \mathrm{~cm}$ barTrial 1

Trial 2 treatment.

Trial 3

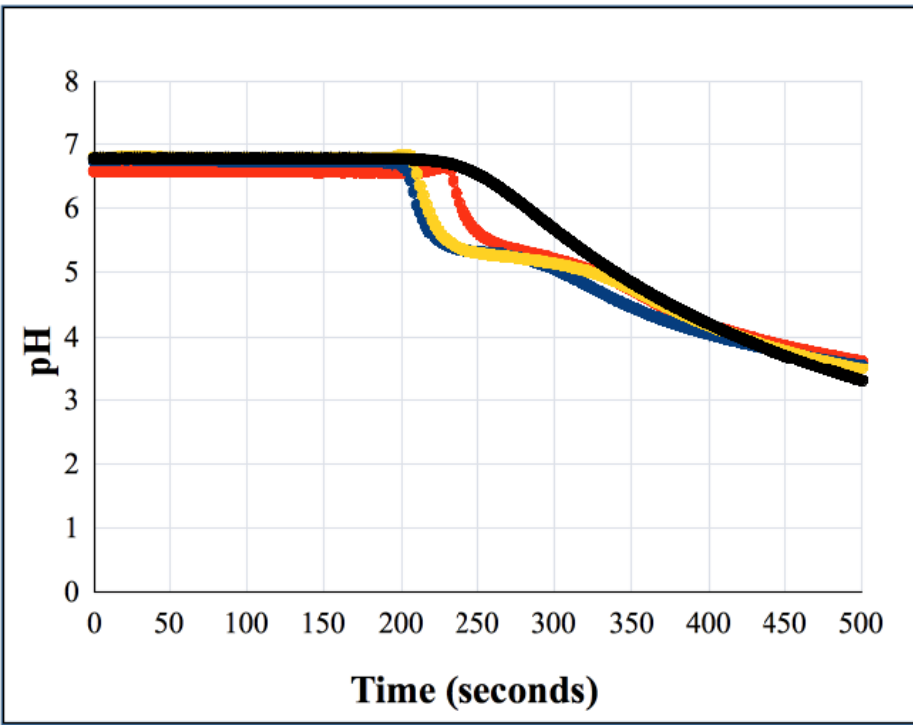

Figure 12: Plot of continuous $\mathrm{pH}$ measurements in two second time intervals in the direct $10.0 \mathrm{~cm}$ barrier-slit treatment with the average fitted model for the Trial 2 treatment.

Trial 3

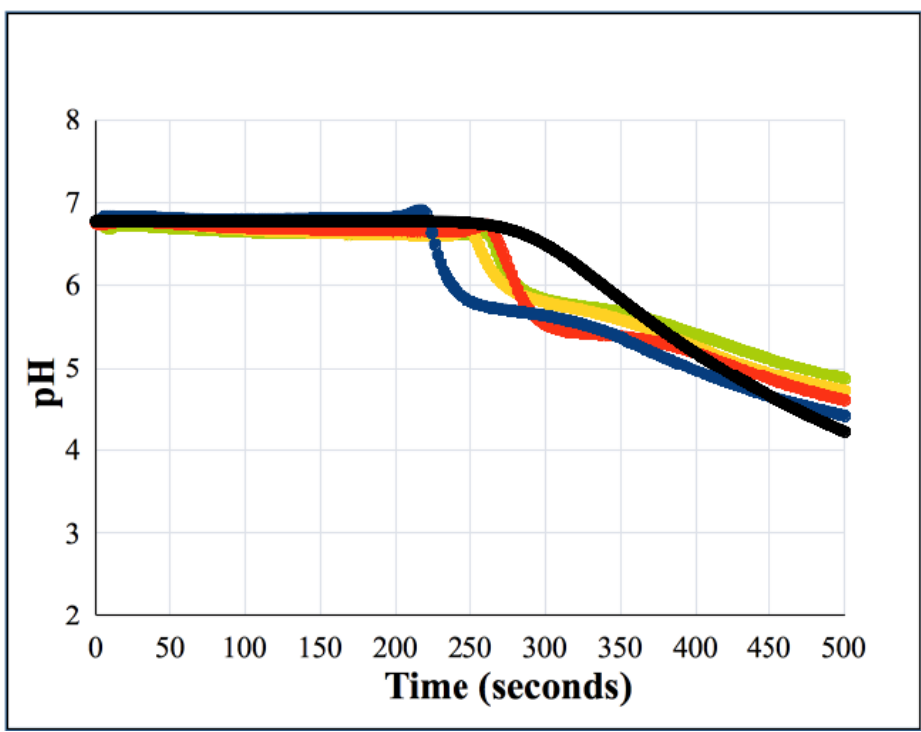

Figure 13: Plot of continuous $\mathrm{pH}$ measurements in two second time intervals in the direct $2.0 \mathrm{~cm}$ barrierslit, shifted $15^{\circ}$ from the central axis, with the average Trial 2 fitted model for the treatment. Trial 3 Trial 4 


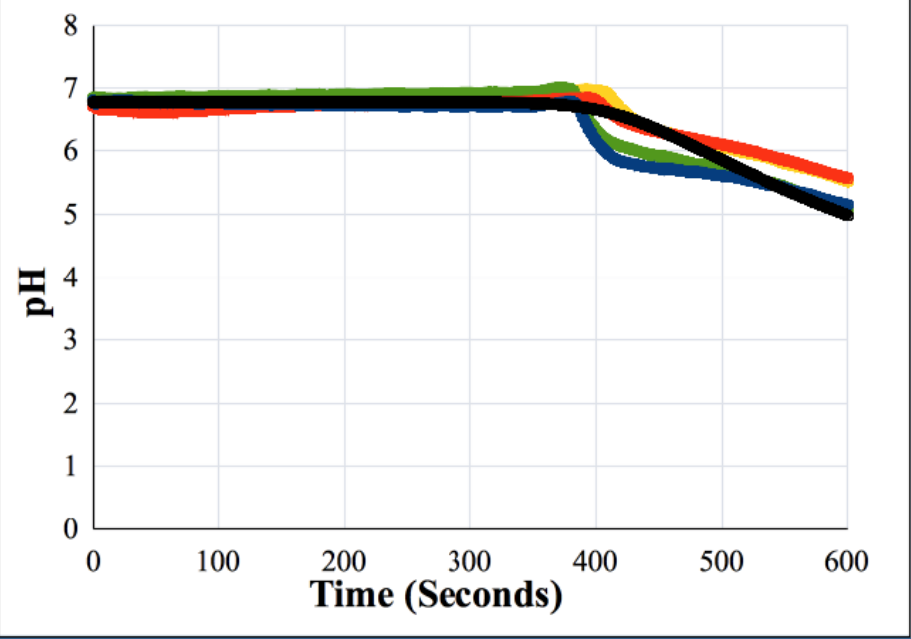

Model

Trial 1

Trial 2

slit, shifted $35^{\circ}$ from the central axis, with the average

Figure 14: Plot of continuous $\mathrm{pH}$ measurements in

two second time intervals in the direct $2.0 \mathrm{~cm}$ barrier-

Trial 3

Trial 4

To determine whether or not the pre-exponential factor varies with obstacle size, for each treatment, the optimized $A$ values of the fitted model functions were averaged and a standard deviation was determined to quantify the variability in the data. These results are shown in Table 2.

Table 2: Mean A values of fitted model functions to the measurements, with standard deviations.

\begin{tabular}{llr}
\hline Treatment & A & $d A$ \\
\hline (1) $0 \mathrm{~cm}$ (no slit) & N/A & N/A \\
(2) $21.2 \mathrm{~cm}$ (no barrier) & 5.0 & 1.4 \\
(3) $1.0 \mathrm{~cm}$ slit & 4.3 & 3.5 \\
(4) $2.0 \mathrm{~cm}$ slit & 4.1 & 3.0 \\
(5) $4.0 \mathrm{~cm}$ slit & 3.3 & 1.2 \\
(6) $10.0 \mathrm{~cm}$ slit & 3.7 & 0.6 \\
(7) $2.0 \mathrm{~cm}$ slit, $15.00^{\circ}$ shift & 3.5 & 1.7 \\
(8) $2.0 \mathrm{~cm}$ slit, $35^{\circ}$ shift & 4.5 & 1.7 \\
Average & 3.9 & 1.0 \\
\hline
\end{tabular}

Although there is no clear trend in A values as the size of the slit increased, the uncertainty in A did increase as the slit became more narrow, which could be accredited to greater stochastic influ- ence. Nevertheless, it was found that all of the comparisons in A values result in a t-score less than the conventional threshold of 1. This indicates that all the pre-exponential factors that the model produced are statistically similar, further validating the method of using macroscopic models of diffusion such as Fick's second law to numerically analyze diffusion scenarios. The exact t-scores are tabulated in the appendix for reference.

\section{Discussion}

An accurate model would predict identical $A$ values between experimental trials because it should account for all variants it attempts to model. Overall, the model gives an excellent approximation for the experimental conditions that were tested, indicated by the statistically similar A values (Table 2). Among all treatments, the average pre-exponential value was found to be $3.9 \pm 1.0$.

Although the t-scores are below the conventional threshold of 1 , it is important to note that this is in part due to the large uncertainties associated with the results, which was calculated based on the variance between experimental trials. Although there is some predicted random uncertainty in this experiment, the presence of large uncertainties suggests there could be systematic errors with either the experimental procedure or with the $\mathrm{pH}$ probe's precision. This indicates that more data sets should be collected to potentially detect outliers in the original data sets.

Despite the study's constraints, the summation of all reasonable paths of diffusion in a system is a reasonable model for twodimensional physically constrained sysems of diffusion. An exciting implication of this discovery is that the general approach of taking the summation of equally probabilistic angles of movement proves to be a reasonable methodology in determining the expected concentration change over time due to diffusion. These positive results provide a further link between Fick's macroscopic description of diffusion and the microscopic properties of diffusion.

While the statistical tests implied a close correlation between the experimental data and the numerical results, it did not escape our attention that certain discrepancies exist. For example, the model predicts the initial pH drop to occur gradually, whereas there was an initial sudden drop in almost all of the experimental measurements. This discrepancy can be attributed to the reflection of acid particles off of the back wall of the arena. An important assumption in Fick's laws is that the arena is infinitely wide. In other words, the agent inducing concentration change cannot reflect back to the $\mathrm{pH}$ probe. It is probable that the momentary sudden drop in $\mathrm{pH}$ observed for most experimental trials is due to the combined effects of both the incoming acid solute from the source and the reflection off of the 
back wall. This explanation is supported by the observation that coloured dye reflects off of the walls upon contact. In order to refine the experimental procedure to comply with the theoretical assumptions of the model it would be necessary to use a large arena to minimize reflections.

Furthermore, another point of conflict between the data and the computational results is the differences in their final slopes. In general, the model predicts a steeper drop in $\mathrm{pH}$ than the actual data after 500 seconds. This is likely due to acid particles lingering in the side of the arena where the insertion container is, and not diffusing to the other half of the arena where the $\mathrm{pH}$ probe is. This suggests a potential area for improvement as our computational model assumes that acid particles will not become trapped in one side of the arena.

Finally, an interesting observation to note is that the $\mathrm{pH}$ in the experimental data sets occasionally in-creases slightly right before it drops. This is likely a result of systematic error since no base was added to the arena. Furthermore, no appreciable side reactions could have occurred between water and $\mathrm{HCl}$ to result in an increase in $\mathrm{pH}$. Therefore, this particular discrepancy can be accredited to the sensitiveness of the $\mathrm{pH}$ probes and any potential mistakes in equipment handling.

Interestingly, if one were to approach the diffusion problem as a solution to the root mean square displacement relationship, as described bv

$$
r^{2}=2 D t
$$

where $r$ is the distance travelled by the hydronium ion, $D$ is its diffusion constant, which was earlier found to be $2.94 \times 10^{-9} \mathrm{~m}^{2} / \mathrm{s}$, and $t$ is the time that it was allowed to diffuse for, then one would find that a hydronium ion would travel less than $2 \mathrm{~mm}$ in $500 \mathrm{sec}-$ onds (2). Although this theoretical expectation appears to be in stark contrast with the proposed diffusion model, where acid particles were able to reach a pH probe over $25 \mathrm{~cm}$ away in approximately 200 seconds, it should be noted that there are three main possible factors responsible for this apparent discrepancy.

Firstly, Equation 8 is the solution for the root mean square displacement of a particle. It does not represent the maximum displacement by any particle. The distribution curve of a particle displacement can be modelled as a Gaussian curve as shown in Figure 15. Although the root mean square displacement is small, there are still many particles which could have travelled a long distance. Since 0.0862 moles of hydrochloric acid were added to the arena containing $700 \mathrm{~mL}$ of water, and only about $7.0 \times 10^{-6}$ moles were required to change the $\mathrm{pH}$ in the arena from 7 to 5 , thus it is highly likely that the $\mathrm{pH}$ changes detected by the probe were due to the relatively few hydronium particles which had high displacement.

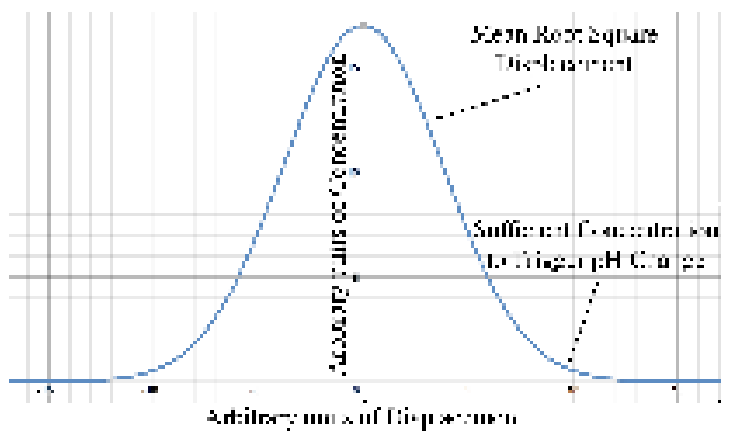

Figure 15: Example of a Gaussian diffusion within a labelled root mean square displacement along with a minimal concentration threshold to trigger a $\mathrm{pH}$ change.

All values are arbitrary.

Additionally, it is possible that the hydronium particles themselves were not diffusing to the $\mathrm{pH}$ probe, but rather it was the diffusion of charge. If the charge gradient between the source and the detector was great enough, it is possible that the change in $\mathrm{pH}$ was induced by positive charges moving from the source to the detector, or more precisely, the movement of electrons from the detector to the source facilitated by the rapid breakage and formation of the $\mathrm{O}-\mathrm{H}$ bonds in water and hydronium, as shown in Figure 16.

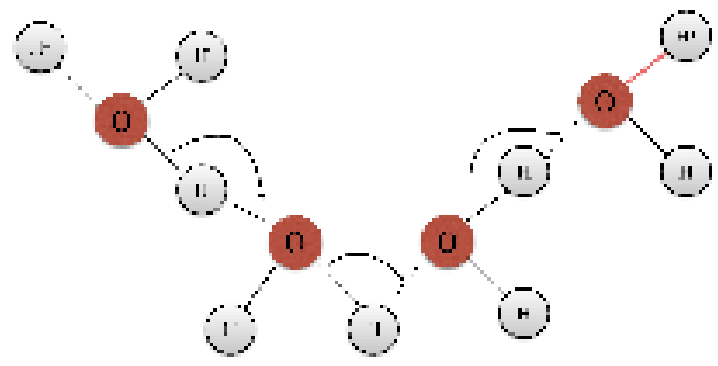

Figure 16: Representation of charge flow with the breaking and formation of $\mathrm{O}-\mathrm{H}$ bonds of water and hydronium..

In addition to these theoretical explanations, systematic errors could also be responsible for such a discrepancy. An inevitable error in the experiments was the initial velocity of the acid particles. When the lid of the container of acid was opened, it could be justifiably assumed that this action transfers some kinetic energy among both the water and acid molecules, which in turn may have accelerated the diffusion process to a certain degree. 


\section{Conclusion}

Despite these sources of error, the model is able to determine that the pre-exponential factor A remains statistically constant as the physical environment of the system changes, which is expected based on pre-established theory $(1 ; 3)$. Among all treatments, the average pre-exponential value was found to be $3.9 \pm 1.0$.

The relative consistency of this model in multiple scenarios suggests that such a combination of numerical integration and Fick's laws could be applied to more complex systems with numerous compartments and geometries, which is a potential direction for future research. The immense applicable aspects of such a model could be used to estimate macroscopic effects given the point of diffusion and the nature of the system. Conversely, it can predict the location of such a point based on the macroscopic effects. From a broader perspective, any physical system of diffusion could be modelled using this approach, albeit the numerical integration would become increasing complex for three-dimensional systems where reflection must be taken into consideration.

\section{Acknowledgements}

We would like to express our cordial gratitude towards Dr. Chris Addison, Director of UBC Science One, for providing equipment, and his valuable insight which greatly assisted the research. In addition, we thank Dr. Elliott Burnell and Dr. Michael Gilbert for sharing with us their opinions on the paper and offering us their valuable insight. Finally, we would like to thank Christina Cheung and Michelle Marcus for reviewing an earlier version of our manuscript and offering comments which greatly improved the quality of the paper.

\section{Appendix}

Table 1: Chi Square and probability values achieved for fitting the model to each experimental trial.

\begin{tabular}{|c|c|c|}
\hline Treatment & Weighted Chi-Square & Probability Value \\
\hline (1) $0 \mathrm{~cm}$ (no slit) & $\mathrm{N} / \mathrm{A}$ & N/A \\
\hline \multicolumn{3}{|l|}{ (2) $21.2 \mathrm{~cm}$ (no barrier) } \\
\hline Trial 1 & 5.8 & $83.4 \%$ \\
\hline Trial 2 & 3.1 & $97.9 \%$ \\
\hline Trial 3 & 1.9 & $99.7 \%$ \\
\hline Trial 4 & 1.8 & $99.8 \%$ \\
\hline \multicolumn{3}{|l|}{ (3) $1.0 \mathrm{~cm}$ slit } \\
\hline Trial 1 & 2.0 & $99.7 \%$ \\
\hline Trial 2 & .7 & $99.8 \%$ \\
\hline Trial 3 & 0.6 & $99.9 \%$ \\
\hline \multicolumn{3}{|l|}{ (4) $2.0 \mathrm{~cm}$ slit } \\
\hline Trial 1 & 1.3 & $99.9 \%$ \\
\hline Trial 2 & 1.9 & $99.7 \%$ \\
\hline Trial 3 & 5.7 & $84.0 \%$ \\
\hline Trial 4 & 2.7 & $98.8 \%$ \\
\hline \multicolumn{3}{|l|}{ (5) $4.0 \mathrm{~cm}$ slit } \\
\hline Trial 1 & 2.3 & $99.3 \%$ \\
\hline Trial 2 & 3.3 & $97.3 \%$ \\
\hline Trial 3 & 2.9 & $98.4 \%$ \\
\hline \multicolumn{3}{|l|}{ (6) $10.0 \mathrm{~cm}$ slit } \\
\hline Trial 1 & 1.8 & $98.4 \%$ \\
\hline Trial 2 & 3.0 & $96.4 \%$ \\
\hline Trial 3 & 3.3 & $95.1 \%$ \\
\hline (7) $2.0 \mathrm{~cm}$ slit, $15.00^{\circ}$ angle shift & 3.5 & 1.7 \\
\hline Trial 1 & 1.8 & $99.8 \%$ \\
\hline Trial 2 & 1.3 & $99.9 \%$ \\
\hline Trial 3 & 0.9 & $99.9 \%$ \\
\hline Trial 4 & 1.2 & $99.9 \%$ \\
\hline (8) $2.0 \mathrm{~cm}$ slit, $35^{\circ}$ angle shift & 4.5 & 1.7 \\
\hline Trial 1 & 2.7 & $99.7 \%$ \\
\hline Trial 2 & 2.8 & $99.7 \%$ \\
\hline Trial 3 & 2.6 & $99.8 \%$ \\
\hline Trial 4 & 1.6 & $99.9 \%$ \\
\hline
\end{tabular}

Table 2: T scores comparing fitted A values between experimental treatments.

\begin{tabular}{|l||l|l|l|l|l|l|l|l|}
\hline Treatment & $\mathbf{1}$ & $\mathbf{2}$ & $\mathbf{3}$ & $\mathbf{4}$ & $\mathbf{5}$ & $\mathbf{6}$ & $\mathbf{7}$ & $\mathbf{8}$ \\
\hline $\mathbf{1}$ & N/A & N/A & N/A & N/A & N/A & N/A & N/A & N/A \\
\hline $\mathbf{2}$ & N/A & 0.0 & 0.19 & 0.27 & 0.92 & 0.85 & 0.68 & 0.23 \\
\hline $\mathbf{3}$ & N/A & 0.19 & 0.0 & 0.043 & 0.27 & 0.19 & 0.21 & 0.051 \\
\hline $\mathbf{4}$ & N/A & 0.27 & 0.043 & 0.0 & 0.25 & 0.13 & 0.17 & 0.12 \\
\hline $\mathbf{5}$ & N/A & 0.92 & 0.27 & 0.25 & 0.0 & 0.30 & 0.10 & 0.58 \\
\hline $\mathbf{6}$ & N/A & 0.85 & 0.17 & 0.13 & 0.30 & 0.0 & 0.11 & 0.44 \\
\hline $\mathbf{7}$ & N/A & 0.68 & 0.21 & 0.17 & 0.10 & 0.11 & 0.0 & 0.42 \\
\hline $\mathbf{8}$ & N/A & 0.23 & 0.51 & 0.12 & 0.58 & 0.44 & 0.42 & 0.0 \\
\hline
\end{tabular}

\section{References}

1. Fick A. On liquid diffusion. J. Membr. Sci. 1995;100(1):33-38.

2. Einstein A. Investigations on the theory of Brownian Movement. New York, N.Y.: Dover Publications; c1956.

3. Crank J. The Mathematics of Diffusion. Oxford: Clarendon Press; c1967.

4. Edward J. Molecular Volumes and the Stokes-Einstein Equation. J Chem Educ. 1970;47(4):261.

5. Haynes WM. CRC Handbook of Chemistry and Physics. Boca Raton, Fla.: CRC Press; c2016. 


\section{REVIEW}

by Dr. James Charbonneau,

\section{Department of Physics and Astronomy, University of British Columbia. Vancouver}

In this paper the authors venture to do three things 1) create numerical model for diffusion in 2-dimensions that can account for diffusion around barriers 2) experimentally verify their numerical model and 3) experimentally verify some standard results that arise from Fick's laws of diffusion. This is particularly notable as it approaches the problems from theoretical, computational, and experimental sides, demonstrating a complete understanding of the topic.

The paper mostly succeeds at its goals, and is of very good quality. The numerical model developed is interesting, the experiment is meticulously carried out, and the statistical analysis is thorough. However, there are some points that I would like to address.

The following is a list of my comments mostly as they appear in the paper, and not in order of importance.

1. As a relatively minor point, at the end of the introduction, and the end of the paper, the authors feel the need to point out applications in a very specific field, approximation of leaks in hydraulic systems. This feels forced. They shouldn't feel obligated to include such narrow justifications. The work stands on its own in a much broader context.

2. The authors often use the word idealized in the introduction and beginning of the methodology when describing the solutions to Fick's law, without describing what idealizations are actually made in these solutions. Defining this would be helpful as their study involves a non-idealized system. Providing the reader a contrast would greatly help understanding exactly what is special about their study.

3. The authors propose a microscopic model for diffusion that involves looking at paths from one point to another with a single bounce. The contribution of each path to the total diffusion is weighted by the angle of the path to a point along the line of the barrier and the total distance that the particle has to travel. This results in particles that take paths to the far edge of a slit are weighted less. It is interesting model, and maybe somewhat intuitive, but the authors never provide physical reasoning for why they would do this. When coming up with a model to describe a physical system, one should always have a physical justification for the assumptions made in the model. There's likely a good reason why their model works, but the authors never state it. The next point addresses this.

4. The idea of reasonable and unreasonable paths int he development of the model is interesting. A key point of their work is choose a subset of possible diffusion paths to represent all the possible paths of diffusion. Those paths in which the particle bounces once, at the line of the boundary, are deemed reasonable, whereas a path in which the particle takes many bounces to get to the final point is unreasonable.

There are two issues here. First, when discussing why they discard unreasonable paths, their justification is that the macroscopic model accounts for all flow, rather than specific paths. This isn't a valid argument. The authors should justify discarding unreasonable paths be-cause they're statistically unlikely, which uses ideas in the microscopic paradigm in which their model lives.

The second issue is that the choice of reasonable paths seems to include a very specific subset of one-bounce paths, those that occur exactly along the line of the boundary. I agree that one bounce paths probably contribute the most to diffusion around the barrier, something which the authors should spend much more time justifying in their paper. However, there are many other two bounce paths where the bounce doesn't happen exactly on the boundary that should be considered. It could be that this deficiency might explain why their model doesn't capture the sudden drop in $\mathrm{pH}$ seen in all their trials, Figures 7-14. Their model predicts a gradual diffusion rate rather that the steep one because it's missing possible paths. Luckily, it think it's the exponential weighting of the path length ri that leads to the success of the model. This should be fleshed out.

5. The experimental procedure is very well described. The inclusion of positive and negative controls is very important. Additionally, in the presentation of the experimental results the authors are careful to use both p-values and t-scores to interpret their data. This makes the conclusion that the A values all agree with each other, which agrees with the theory, very 
strong. With the increasing concern of the incorrect usage of p-values, this is a very, very good. You should always use multiple statistics to interpret your data to ensure you're not being fooled, or fooling yourself.

6. The plots of the numerical model to the experimental data, Figures 7-14 are very interesting. We see there are many differences between the numerical model and the experimental results. Three things of note: 1) in some experimental trials, the $\mathrm{pH}$ seems to slightly spike before it drops, which the model never does, 2) The $\mathrm{pH}$ drops much faster than the model, and 3) the final slope of the trails is very different than the model.

In the discussion the authors comment on the second of these, speculating that the there are errors within the $\mathrm{pH}$ probe, their model, or the experimental setup. This is a scattershot approach to cover their bases, and seem somewhat contrived. The authors should have used this as a opportunity to critique the underlying assumptions of their computational model.

7. In the discussion the authors include a very nice description of why their results don't match with a pure root-mean-square diffusion calculation.

Overall the paper is very enjoyable and an outstanding example of the interplay between physical theory, mathematical modelling, and experimental evidence. Well done.

One thing that is missing is an attempt to use their computational model to calculate the pre-exponential value A. The analysis involving the computational model in general only involves a qualitative comparison with experimental data. The study would be greatly strengthened if the authors could make a quantitative comparison between model and data. Pointing out that they look similar doesn't tell us as much. 